defined objective: to support the Federal Government in effectively stabilising the Somali state and reconstructing a functioning rule of law. By purposely involving the region and Somalia itself in the process of stabilising the Horn of Africa, the Somalis can re-build their country and re-grow from the inside instead of having to operate in an imposed administrative and authoritative structure. In this respect, it is crucial to pay due attention to the prevalent clan structure and radical forces in Somalia, including al-Shabaab. This might be the only way out of the internationalisation of the Somali crises, which has plagued the state at the Horn of Africa for decades and has not lead to a sustainable cure for the core illness of Somalia. Counterproductive involvement by states such as Eritrea, supremacy struggles, and national interests must be accounted for when involving regional powers side by side and must be kept to the minimum to allow for a sustainable solution focused on Somalia's wellbeing.

The role of European and other international forces should mainly be a supportive one. With their experience and expertise they can support Somali and regional forces in enabling regional ownership. Their main area of involvement, however, should remain the fight against the symptoms, terrorism and piracy, which they have been tackling rather successfully, also with the help of regional powers. This division between one coherent regional/local approach to the core problems and international help with regard to the symptoms will not only clearly define mandates, duties and activities, but it will also offer more specific and defined goals to the missions, resulting in a greater likelihood for success.
Additional recommendations include the involvement of the Arab League in the process of stabilising Somalia, as well as of countries bordering the maritime areas that are affected by Somali piracy and hence have a great interest in the stabilisation of the region. Furthermore, in any kind of consideration on how to amend the situation in Somalia, it is essential to pay due attention to the Somali diaspora living abroad. They play a key role within the context of both piracy and terrorism, not only as possible supporters of both criminal activities, but also as driving forces for a successful rebuilding and stabilisation of their country. Thus, the diaspora should be included in any kind of stabilisation process in Somalia.

The recent passing of a constitution in June 2012 and the swearing in of a new parliament and president in August and September 2012 have certainly been first steps towards such a development. It remains to be seen whether the new Federal Government will be able to successfully tackle the root causes of Somalia's instability and to what extent the Somali population will, after years of anarchy, allow the government to rule. Its achievements in catering basic needs related to food, income, social stability, justice, security, education, etc. will determine its own success in reconciling and reuniting the country. This will be especially interesting with regard to the regions of Somaliland and Puntland that are striving for more autonomy. In any case, honest and committed international as well as regional involvement will remain of great importance in guaranteeing Somalia's successful future.

\title{
Wo steht die Afrikanische Friedens- und Sicherheitsarchitektur? Bilanz und Herausforderungen
}

\author{
Judith Vorrath*
}

\begin{abstract}
When considering the areas of policy formulation, institution-building and actual peace-related activities, the African Peace and Security Architecture (APSA) has overall made substantial progress. Because of APSA's continuing strong external dependence, capacity-building remains necessary. However, it must balance the entire structure; furthermore, by itself it will not be sufficient to guarantee "African ownership". The future of APSA is not simply a question of money or the adequate implementation of projects, but it requires the involvement of civil society as well as a clear political and conceptual framework for "African" solutions. It is crucial for the AU under the new Commission Chairwoman to devise and focus on its own political approaches, a balanced institutional structure, and a strengthening of the multi-dimensional profile of its operations..
\end{abstract}

Keywords: African Union, peace, security, regional organization, peace-building

Afrikanische Union, Frieden, Sicherheit, Regionalorganisation, Peacebuilding

\section{Einleitung}

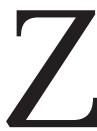
um Zeitpunkt der Gründung der Afrikanischen Union (AU) vor zehn Jahren schienen die Aussichten der Organisation, Frieden und Sicherheit auf dem Kontinent eigenständig und nachhaltig zu stärken, relativ gering. In einer Vielzahl von Mitgliedstaaten waren in den 1990er
Jahren innerstaatliche Konflikte ausgebrochen oder erneut eskaliert und legten die weitgehende Wirkungslosigkeit der Organisation für Afrikanische Einheit (Organisation of African Unity, OAU) als AU-Vorgängerin bei der Prävention, dem

\footnotetext{
Dr. Judith Vorrath ist Wissenschaftlerin in der Forschungsgruppe Sicherheitspolitik der Stiftung Wissenschaft und Politik (SWP), Berlin. Die Autorin dankt Claudia Simons für ihre Anregungen zu diesem Artikel, besonders zur Frage von "African ownership“.
} 
Management und der Lösung von Konflikten schonungslos offen. Die AU sollte einen Neuanfang markieren, doch war dieser mit einigen Hypotheken belastet: Zum einen orientierte sich die AU weiterhin an Prinzipien wie der territorialen Integrität und der Nichteinmischung, deren weitgehende Unantastbarkeit die OAU in der Vergangenheit oft gelähmt hatte. Zum anderen spiegelte die Tatsache, dass mit Ausnahme Marokkos alle Staaten des afrikanischen Kontinents Mitglieder der AU wurden, zwar das Ideal afrikanischer Solidarität wider, stellte aber gleichzeitig jeder wegweisenden Beschlussfassung die Aushandlung unter $53^{1}$ Staats- und Regierungschefs voraus, von denen zudem nicht wenige an der Spitze politisch und ökonomisch fragiler Staaten standen. Auch war die Rolle des AU-Initiators Muammar al-Gaddafi, der neben anderen Persönlichkeiten die Idee der „Vereinigten Staaten von Afrika" propagierte und erhebliche Mittel für den neuen Staatenbund bereitstellte, umstritten und führte immer wieder zu Zerreißproben zwischen den Mitgliedstaaten.

Vor diesem Hintergrund hat die AU bis heute unumstritten wichtige Erfolge erzielt. Das Kerngerüst der Afrikanischen Friedens- und Sicherheitsarchitektur (African Peace and Security Architecture, APSA) ist mit dem 2004 errichteten Friedens- und Sicherheitsrat (Peace and Security Council, PSC) als zentralem, kollektivem Entscheidungsorgan sowie der Abteilung für Frieden und Sicherheit in der AU-Kommission fest etabliert. Bis September 2012 hat der Rat insgesamt 335 Sitzungen abgehalten und dabei eine Vielzahl von Bedrohungen für Frieden und Sicherheit auf dem Kontinent debattiert und entsprechende Beschlüsse gefasst. Neben dem Aufbau weiterer Säulen innerhalb der APSA wurden diplomatische Initiativen und Vermittlungsversuche sowie AU-Friedensmissionen mandatiert. Zudem verurteilte und sanktionierte der PSC verfassungswidrige Regierungswechsel, wie zuletzt Anfang 2012 in Guinea-Bissau und Mali.

Eine systematische Bilanz der APSA muss sich aber jenseits ihrer reinen Funktionalität auch an den gesteckten Zielen und Hoffnungen der Gründungszeit orientieren. Der Leitsatz „afrikanische Lösungen für afrikanische Probleme" steht dabei sinnbildlich für die innere und äußere Erwartungshaltung an die AU.

\section{Mehr Frieden durch afrikanische Lösungen?}

Von Beginn an wurde die APSA als der Inbegriff afrikanischer Eigenverantwortung und „ownership“ verstanden, auf dem Kontinent und darüber hinaus. Dieses Ideal leitet sich unter anderem aus Arbeiten afrikanischer Vordenker wie dem in Kenia geborenen Autor und Wissenschaftler Ali Mazrui ab. Schon 1967 konstatierte er in seiner Arbeit „Towards a Pax Africana“, dass Afrikas Freiheit von Fremdbestimmung davon abhängen wird, ob es in der Lage sei, sich selbst zu befrieden. Regionalorganisationen wie die AU gelten als adäquates Instrument zur Einlösung dieses Anspruches. Die elementare Grundannahme dabei war und ist, dass solche Organisationen komparative Vorteile bei der Konfliktprävention und -lösung in ihrem Einflussbereich besitzen.

1 Mit der Unabhängigkeit des Südsudans im Jahr 2011 stieg die Zahl auf 54 Staaten.
Grundsätzlich ist die zentrale Rolle von Regionalorganisationen beim Erhalt und der (Wieder)Herstellung von Frieden international anerkannt, so etwa in der Agenda für den Frieden der Vereinten Nationen (VN) von 1992. Aus Sicht vieler Experten wie politischer Entscheidungsträger drängt sich eine so verstandene stärkere Rolle von Regionalorganisationen geradezu auf, da die Mehrheit der bewaffneten Konflikte transnationale Dynamiken aufweist und regional verflochten ist. Regionale Ansätze bieten aus dieser Sicht den idealen Rahmen zur Konfliktlösung, da Anrainerstaaten direkter betroffen sind und somit einerseits besser über die Konfliktursachen und -zusammenhänge informiert sind, andererseits stärkere Anreize haben, sich bei der Konfliktlösung zu engagieren. Zudem erhalten Regionalorganisationen wie die AU ihr Mandat nicht „Von oben“ (sprich: von den VN), sondern „von unten und von innen" (sprich: von den kooperierenden Staaten und regionalen Zivilgesellschaften). ${ }^{2}$ Sie werden also wegen der Nähe und stärkeren Bindung als effizienter eingestuft als „weiter entfernte" Organisationen wie die VN. Sie stehen zwischen der internationalen und der lokalen Ebene, die oftmals ein sehr unterschiedliches Verständnis von Peacebuilding haben. Die kritische Literatur räumt daher Regionalorganisationen zumindest das Potenzial ein, den liberalen Peacebuilding-Ansatz, wie ihn die VN und Bretton-Woods-Organisationen verfolgen, herauszufordern. ${ }^{3}$

Diese Annahmen spiegeln sich in den Erwartungen an die APSA wider. Geografische und kulturelle Nähe, eine engere Anbindung an lokale Vorstellungen und Verhältnisse sowie regionale Verflechtungen sollen Triebfedern für eine stärkere Eigenständigkeit sein und versprechen vermeintlich bessere Ergebnisse als das Engagement externer Akteure. Dabei wird der AU als „genuin afrikanischer" Organisation die Hauptrolle als Friedensstifterin und -bewahrerin auf dem Kontinent zugedacht. Inwiefern konnte sie diese Rolle seit ihrer Gründung ausfüllen? Und wo liegen die besonderen Herausforderungen für die APSA in den nächsten Jahren? Diesen Fragen wird nachfolgend mit Blick auf die konzeptionelle, strukturelle und operative Entwicklung der APSA nachgegangen.

\section{Neue Politik, alte Konzepte}

Anders als die OAU ist die AU gemäß ihrer Doktrin für die Bearbeitung interner Konflikte aufgestellt und verfolgt einen klar interventionistischeren Ansatz. Zwar wurde schon durch die OAU 1993 der Mechanismus für Konfliktprävention, -management und -lösung entwickelt. Inhaltlich durchaus ein Meilenstein, blieb dieser aber real weitgehend wirkungslos. Dem daraus resultierenden Vorwurf der Gleichgültigkeit gegenüber innerstaatlichen Konflikten begegnete die AU in ihrer Gründungsakte von 2000 mit der Verankerung des Ansatzes der „non-indifference“. Artikel 4(h) sah, zum ersten Mal für eine Organisation, die für die friedliche Regelung und Lösung

2 Hettne, Björn/Söderbaum, Fredrik. 2006. The UN and Regional Organizations in Global Security: Competing or Complementary Logics? Global Governance, 12, S. 227-232, S. 229.

3 Siehe beispielsweise: Lidén, Kristoffer/Mac Ginty, Roger/Richmond, Oliver P. 2009. Introduction: Beyond Northern Epistemologies of Peace: Peacebuilding Reconstructed? International Peacekeeping, 16: 5, S. 587598. 
von Konflikten unter ihren Mitgliedstaaten verantwortlich ist, vertraglich das Recht auf Intervention auch unabhängig von der Zustimmung des betreffenden Mitgliedstaates vor. ${ }^{4} \mathrm{Da}-$ mit ging die AU schon fünf Jahre vor dem Weltgipfel der VN, auf dem das Prinzip der Schutzverantwortung angenommen wurde, neue Wege.

Daneben stützt sich die AU auf einen umfassenden, multidimensionalen Ansatz von Frieden und Sicherheit. Entsprechend gehört auch das Konzept des Peacebuilding zu ihrem Repertoire, das in Artikel 14 des Protokolls über die Einsetzung des Friedens- und Sicherheitsrates (PSC-Protokoll) von 2002 spezifiziert ist. Dort werden Maßnahmen wie die Konsolidierung von Friedensabkommen, die Schaffung von Bedingungen für einen politischen, sozialen und ökonomischen Wiederaufbau und die (Wieder)Herstellung von Rechtstaatlichkeit und demokratischen Institutionen genannt. ${ }^{5}$ Das Leitbild der menschlichen Sicherheit wurde zudem später in der Gemeinsamen Afrikanischen Verteidigungs- und Sicherheitspolitik von 2004 verankert. Damit wird nicht nur die Bedeutung politischer Werte, sondern auch sozialer und ökonomischer Bedürfnisse anerkannt. ${ }^{6}$ Konkretisiert wird dieser umfassende Ansatz in Dokumenten wie der "Policy on Post-Conflict Reconstruction and Development (PCRD)", die ein Drei-Phasen-Modell vorsieht mit kurzfristiger Stabilisierung, mittelfristig gefolgt von einer Übergangsphase und einer längerfristigen Phase nachhaltiger Entwicklung zur Förderung von Versöhnung und sozio-ökonomischem Wiederaufbau. ${ }^{7}$ Grundsätzlich erkennt die AU damit die Beziehung zwischen Frieden, Sicherheit und Entwicklung an und fordert gleichzeitig afrikanische Eigenständigkeit und Führung.

Konzeptionell allerdings unterscheidet sich das Verständnis von Peacebuilding in Dokumenten wie dem PSC-Protokoll kaum von dem internationaler Berichte wie der "Agenda für den Frieden" oder dem späteren Bericht „In größerer Freiheit" des damaligen VN-Generalsekretärs Kofi Annan. Insgesamt leitet sich der AU-Ansatz weitgehend aus dem liberalen Friedensmodell her. Während also einerseits „eine afrikanische Vision der Erneuerung und nachhaltigen Entwicklung"8 propagiert wird, fügt sich die Politikformulierung der AU in den globalen Rahmen ein und ist darauf ausgerichtet, diesen vor allem in Kooperation mit den VN zu ergänzen. Neue Ansätze der Konfliktprävention oder der Friedenskonsolidierung, die sich substanziell vom westlich geprägten und oftmals kritisch bewerteten liberalen Konzept abheben, sucht man in AU-Dokumenten weitgehend vergebens.

So stark die AU im ersten Jahrzehnt ihres Bestehens in der Politikformulierung allgemein war, so wenig hat eine konzeptionelle Weiterentwicklung von „afrikanischen Lösungen“ stattgefunden. Dies mag damit zu tun haben, dass die AU weiterhin vor allem „top-down“ und staatszentriert funktioniert

4 Slotin, Jenna/Castro Wesamba/Kebede, Teemt. 2009. The Responsibility to Protect (RtoP) and Genocide Prevention in Africa. New York: International Peace Institute.

5 African Union. 2002. Protocol Relating to the Establishment of the Peace and Security Council of the African Union, S. 22.

6 African Union. 2004. Solemn Declaration on a Common African Defence and Security Policy, S. 3.

7 African Union. 2006. Policy on Post-Conflict Reconstruction and Development African Union.

8 Ebd., S. 3. und sich mit der Einbeziehung von Zivilgesellschaften schwer tut. Die Beteiligung von zivilgesellschaftlichen Gruppen ist weitgehend ein Lippenbekenntnis geblieben. ${ }^{9}$ Zwar ist es keineswegs ungewöhnlich, dass komplexe Gebilde wie die APSA Schwierigkeiten haben, innovative Ansätze für ihre Politik zu entwickeln und für Ideen „von unten“ durchlässig zu sein. Doch der komparative Vorteil der AU wird gerade in der engeren Anbindung an „lokale“ Zusammenhänge gesehen. Real wird die Organisation bislang vor allem von afrikanischen Eliten und ihren Interessen und Vorstellungen von Frieden und Sicherheit geprägt, was die Frage aufwirft, wessen „ownership“ gemeint ist, wenn von afrikanischer Eigenständigkeit die Rede ist. Hinzu kommt, dass die APSA institutionell und operativ stark außenabhängig ist.

\section{Institutioneller Aufbau und subsidiäre Schieflage}

Neben dem PSC und der Abteilung für Frieden und Sicherheit der AU-Kommission sieht die APSA weitere institutionelle Säulen vor. Dazu gehören das kontinentale Frühwarnsystem, die afrikanische Eingreiftruppe, zwei beratende Gremien und der Friedensfonds zur Finanzierung operativer Aktivitäten. Die geplante Eingreiftruppe mit Brigaden in den fünf Regionen Nord-, West-, Zentral-, Ost- und südliches Afrika ist wohl das ambitionierteste Projekt. Bis 2015 sollen diese Kapazitäten, die militärische wie zivile Komponenten umfassen, einsatzbereit sein. Sie sollen Friedenseinsätze mit afrikanischem Personal unter afrikanischer Führung und ein schnelles Eingreifen besonders bei nach Artikel 4(h) mandatiertem Vorgehen ermöglichen.

Trotz des Fortschritts beim Aufbau dieser Pfeiler ist ihre Funktionalität bislang nicht (voll) gewährleistet. Die Prüfung der potenziellen Einsatzfähigkeit der Eingreiftruppe 2010 und 2011 ergab deutliche Defizite, darunter einen Mangel an Kapazitäten in der Verwaltung, Finanzierung, Kommunikation, personellen Ausstattung und der Planung von Missionen. ${ }^{10}$ Ähnlich ist es beim Frühwarnsystem, das trotz Verbesserungen unter einem Mangel an Expertise und Ausrüstung leidet, und beim PSC-Sekretariat, das seiner Aufgabe, den Rat zu unterstützen und zu ergänzen, wegen fehlenden Personals und fehlender finanzieller Ressourcen nur eingeschränkt gerecht werden kann. In diesem Sinne wurde auch in einem internen AU-Bericht ein fehlender Wille der Mitgliedstaaten unterstellt, zusätzliche Mittel bereitzustellen und damit zur weiteren Operationalisierung der APSA beizutragen. ${ }^{11}$

Neben dem Mangel an Ressourcen und Ausstattung existieren weitere Baustellen, insbesondere im subsidiären Aufbau der APSA. Aufgrund der Annahme, dass eine „Nähe“ zu Krisen- und Konfliktgebieten vorteilhaft für die Effektivität einer Organisation bei der Konfliktlösung ist, fußt die Architektur auf regio-

9 Murithi, Tim. 2010. The African Union Peace and Security Partnerships. In Beyond Development Aid: EU-Africa Political Dialogue on Global Issues of Common Concern, edited by EARN: Europe Africa Policy Research Network (EARN). Drafted November 2010, S. 52.

10 Bachmann, Olaf. 2011. The African Standby Force: External Support to an 'African Solution to African Problems'? Brighton: Institute of Development Studies. Research Report Vol. 2011 No. 67, April 2011, S. 13

11 African Union. 2010. African Peace and Security Architecture (APSA): 2010 Assessment Study, S. 30. 
nalen Pfeilern unterhalb der Ebene der AU. Diese sind vor allem die schon länger bestehenden Regionalen Wirtschaftsgemeinschaften (RECs), die ihrerseits sicherheitsrelevante Strukturen aufgebaut haben. Artikel 16 des Protokolls über die Einsetzung des Friedens- und Sicherheitsrates sieht einerseits die Harmonisierung und Koordinierung mit diesen regionalen Pfeilern durch den Rat sowie die AU-Kommission vor, und andererseits enge Arbeitsbeziehungen und Partnerschaften, deren Modalitäten „Von dem komparativen Vorteil“ des jeweiligen Partners sowie der vorherrschenden Situation bestimmt werden sollen. ${ }^{12}$ Dieses Dokument sowie ein "Memorandum of Understanding", abgeschlossen zwischen der AU und den regionalen Organisationen und Mechanismen im Jahr 2008, bilden die Grundlage dieses Ansatzes. Am deutlichsten zeigt sich dieser im Aufbau der Afrikanischen Eingreiftruppe und des Frühwarnsystems. Die RECs und die Mitgliedstaaten liefern Informationen an das Frühwarnzentrum im AU-Hauptquartier. Zudem sind sie verantwortlich für den Aufbau und die Bereitstellung der regionalen Brigaden. Schon jetzt stützt sich die AU in den Bereichen Konfliktprävention sowie Friedensschaffung, -sicherung und -konsolidierung auf die RECs. In der jüngsten Krise in Mali beispielsweise hat der AU-Friedens- und Sicherheitsrat praktisch alle zentralen Aspekte einer Konfliktlösung an die westafrikanische Wirtschaftsgemeinschaft (ECOWAS) delegiert.

Durch die Einbindung von Organisationen unterhalb der AU ist jedoch eine Schieflage entstanden. Denn der Integrationsprozess ist in den verschiedenen Regionen sehr unterschiedlich fortgeschritten und institutionalisiert. Für den Aufbau der Eingreiftruppe beispielsweise mussten in Nord- und Ostafrika eigens regionale Mechanismen geschaffen werden, während in den anderen Regionen bereits etablierte RECs als Anker dienen konnten. ECOWAS und mit Einschränkungen auch die Südafrikanische Entwicklungsgemeinschaft (SADC) können auf eine relativ gut etablierte Sicherheitsinfrastruktur zurückgreifen, während die Wirtschaftsgemeinschaft der zentralafrikanischen Staaten über weitaus weniger Kapazitäten verfügt. Zudem existieren in Zentral- und Ostafrika mehrere sich überlappende Regionalorganisationen, die offiziell in die APSA eingebunden, deren Friedensbemühungen aber sehr begrenzt sind. Der APSA fehlt damit in diesen Regionen ein wichtiger Anker und Hebel. Mitgliedstaaten der AU greifen besonders hier weiterhin eigenmächtig in Nachbarländern ein, wie in Somalia oder dem Osten der Demokratischen Republik Kongo, obwohl dort AUbzw. VN-Friedensmissionen bestehen. Sie unterminieren damit einen regionalen Multilateralismus und die Funktionalität der APSA, obwohl einige dieser Staaten, z.B. Ruanda und Äthiopien, zu ihren wichtigsten Truppenstellern und Geldgebern gehören.

Es existieren auch weiterhin Unklarheiten im Verhältnis von RECs und AU. In der Roadmap zur Operationalisierung der Eingreiftruppe von 2005 wurde präzisiert, dass die regionalen Wirtschaftsgemeinschaften und Mechanismen Interventionen durch die AU autorisieren lassen sollen. ${ }^{13}$ Diese Vorgabe

12 African Union. 2002. Protocol Relating to the Establishment of the Peace and Security Council of the African Union, S. 23.

13 African Union. 2005. Roadmap for the Operationalization of the African Standby Force. In Experts' Meeting on the Relationship between the AU and the Regional Mechanisms for Conflict Prevention, Management and Resolution. Addis Ababa: African Union. 22-23 March 2005, EXP/AU-RECs/ ASF/4(I), S. 5. ist allerdings umstritten. Organisationen wie ECOWAS und SADC, die sich bereits erfolgreich als Akteure im Bereich Frieden und Sicherheit etabliert haben, erkennen die Führungsfunktion der AU nicht immer an. Hinzu kommen Ängste von Mitgliedstaaten und RECs, dass sie die Kommandogewalt an das AU-Hauptquartier in Addis Abeba verlieren, obwohl sie die Kontingente der Eingreiftruppe stellen.

Insgesamt kann die APSA bei allen verbleibenden Herausforderungen auf eine komplexe und in ihren Grundzügen etablierte Struktur verweisen. Sie setzt sich dabei von institutionellen Vorbildern, etwa den VN, durchaus ab. So gibt es kein Vetorecht und keine permanenten Mitglieder im AU-Friedensund Sicherheitsrat. Blockaden können auf diese Weise verhindert werden. Kriterien für die Mitgliedschaft umfassen unter anderem die Bindung der Staaten an Standards der verantwortungsvollen Regierungsführung, dem Grad der Rechtstaatlichkeit und der Verwirklichung von Menschenrechten sowie an Quoten für die Vertretung der verschiedenen Regionen Afrikas. Das zivile Beratungsgremium der APSA, der Rat der Weisen, ist nach dem Vorbild der traditionellen Ältestenräte in vielen afrikanischen Gesellschaften gestaltet. Fünf hochrangige afrikanische Persönlichkeiten gehören jeweils für drei Jahre diesem Gremium an, das PSC und AU-Kommission zu friedensrelevanten Themen berät. Der Rat soll auch in Konflikten vermitteln und bei der Vorbereitung und Umsetzung von Friedensabkommen helfen sowie Versöhnungsprozesse unterstützen. In diesem Sinne ist er ein „genuin afrikanisches“ Gremium, das allerdings seit seiner Einrichtung 2007 nur sehr begrenzt tätig geworden ist.

\section{Aktiv, aber abhängig}

Trotz des noch unvollständigen institutionellen Aufbaus hat die APSA bereits eine Vielzahl von Aktivitäten hervorgebracht. Als Kernstück der APSA kann der PSC Maßnahmen der präventiven Diplomatie, der Friedensschaffung, -sicherung und -konsolidierung sowie Interventionen nach Artikel 4(h) autorisieren. Während Letzteres bislang noch nicht stattgefunden hat, hat der PSC unter anderem versucht, durch die Ernennung von Sonderbeauftragten und Sondergesandten sowie die Einrichtung von Panels wie dem „High-level Implementation Panel for Sudan" auf Konflikte einzuwirken. Da auch verfassungswidrige Regierungswechsel eine Gefahr für Frieden und Sicherheit darstellen, kann der Rat in solchen Fällen Sanktionen gegen den jeweiligen Mitgliedstaat initiieren. Tatsächlich ist dies bereits wiederholt geschehen, so unter anderem nach den Präsidentschaftswahlen 2010 in der Elfenbeinküste als sich der abgewählte Präsident Laurent Gbagbo weigerte, die Macht abzugeben. Häufiger allerdings verhängt der PSC Sanktionen nach erfolgten Putschen und suspendiert die Mitgliedschaft des betreffenden Landes in der AU. Dies war etwa 2008 der Fall in Guinea, Madagaskar und Mauretanien, 2010 in Niger sowie jüngst in Mali und Guinea-Bissau. Demgegenüber hat der PSC allerdings die Parteien, die an der Gewalt im Zuge der Wahlen in Kenia (2007/8) und in Simbabwe (2008) beteiligt waren, nicht verurteilt. Während Putschversuche also weitgehend geächtet werden, tut sich der Rat damit bei an- 
deren gewaltsamen Disputen um Regierungsmacht schwerer. Dies mag auch damit zusammenhängen, dass die ursprünglich aufgestellten Kriterien für die Mitgliedschaft im PSC ausgehöhlt und Staaten mit fragwürdigem Ruf im Bereich der Regierungsführung und Menschenrechte aufgenommen wurden. ${ }^{14}$

Die größte Aufmerksamkeit haben wohl die AU-Friedensmissionen in Burundi, Sudan/Darfur und Somalia erregt. ${ }^{15}$ Da die AU-Eingreiftruppe noch nicht einsatzbereit ist, musste in diesen Fällen auf die freiwillige Bereitstellung von Truppen durch die Mitgliedstaaten zurückgegriffen werden. Die Operationen basieren nicht auf einer alternativen Vorstellung von Friedensmissionen, sondern spiegeln - jedenfalls auf dem Papier - weitgehend den multidimensionalen Ansatz heutiger VN-Missionen wider. Wohl auch um ihr unabhängiges Profil zu stärken, hat die AU allerdings die kontroverse Regelung getroffen, die VN lediglich über geplante Aktionen zu informieren, ohne notwendigerweise vorab eine Autorisierung des Vorgehens zu erbitten.

Von echter Eigenständigkeit sind die AU-Missionen allerdings weit entfernt. Grundsätzlich besteht eine große Diskrepanz zwischen den Ambitionen der APSA und ihren technischen und finanziellen Ressourcen. Es klafft eine erhebliche Lücke zwischen der Bereitschaft des PSC Friedensmissionen zu autorisieren und den Fähigkeiten der AU diese umzusetzen. ${ }^{16}$ Somit sind AUMissionen bislang stark abhängig von Geldern und operativen Kapazitäten externer Partner, vor allem EU und VN. Zwischen Mitte 2008 und 2012 beispielsweise erhielt die AU-Mission in Somalia (AMISOM) ungefähr 800 Millionen US-Dollar von den VN. ${ }^{17}$ Die EU hat im Rahmen der Friedensfazilität für Afrika insgesamt 600 Millionen Euro für afrikanische Friedensmissionen bereitgestellt, insbesondere zur Deckung operativer Kosten für Transport, Unterkunft, Benzin und Kommunikation. ${ }^{18}$ Die Diskrepanz zwischen Anspruch und Wirklichkeit zeigt sich besonders deutlich beim AU-Friedensfonds. Die AU-Mitgliedstaaten haben hier zwischen 2008 und 2011 nur zwei Prozent der bereitgestellten Mittel beigetragen, während der Rest von internationalen Gebern beigesteuert wurde. ${ }^{19}$

Die mangelnde Verfügbarkeit von eigenen Ressourcen ist auch eine Erklärung dafür, dass sich bei den Missionen eine Art "Arbeitsteilung“ zwischen AU und VN entwickelt hat. Peacebuilding mit zivilen Mitteln, wie es in verschiedenen AU-Dokumenten vorgesehen ist, hat im Rahmen von AU-geführten Missionen kaum stattgefunden. Stattdessen profiliert sich die APSA bislang vor allem mit relativ kurzen und militärisch geprägten Missionen, die faktisch der ersten Phase einer Stabilisierung im Drei-Phasen-Modell der PCRD entsprechen. Dabei enthielten die Mandate aller bisherigen AU-Missionen auch friedenskonsolidierende Elemente wie sie in Artikel 14 des

14 Sturman, Kathryn/Hayatou, Aïssatou. 2010. The Peace and Security Council of the African Union: From Design to Reality, in: Engel, Ulf/ Porto, J. Gomes (Hg.). Africa's New Peace and Security Architecture: Promoting Norms, Institutionalizing Solutions, Farnham: Ashgate, S. 57-75.

15 Darüber hinaus intervenierte die AU 2008 auf den Komoren.

16 African Union. 2010. African Peace and Security Architecture (APSA): 2010 Assessment Study, S. 26

17 Williams, Paul D. 2011. The African Union's Conflict Management Capabilities. New York: Council on Foreign Relations, S. 16.

18 EuropeAid. 2012. AMISOM. European Commission 2012: http://ec.europa. eu/europeaid/where/acp/regional-cooperation/peace/peace-supportoperations/amisom_en.htm [20. Juli 2012].

19 ISS. 2012. Peace and Security Council Report. Institute for Security Studies. Issue 36. Juli 2012.
PSC-Protokolls angelegt sind. Die erste AU-Mission überhaupt, die Mission in Burundi, übergab allerdings bereits nach etwa einem Jahr an die VN, die dann überwiegend die mittel- und längerfristigen Aufgaben zivilen Peacebuildings übernahmen. Im Sudan wurde die 2004 eingesetzte AU-geführte Mission für Darfur Ende 2007 in eine hybride Friedenstruppe, bestehend aus AU und VN, überführt. Auch für die laufende Mission in Somalia war und ist eine Übergabe an die VN grundsätzlich vorgesehen. Das Unterstützungsbüro der VN für AMISOM (United Nations Support Office for AMISOM) soll diese vorbereiten. Allerdings hat die Übergabe bislang aufgrund des schleppenden Fortschritts des Friedensprozesses und der schwierigen (politischen) Rahmenbedingungen noch nicht stattgefunden.

Somit fungieren AU-Missionen bislang als eine Art Wegbereiter für ein längerfristiges und umfassenderes Engagement der VN. Bedenklich ist dabei die Tendenz, Stabilisierungsmaßnahmen in besonders unsicheren Kontexten zunächst an die AU abzutreten, die dann aber bei den mittel- und längerfristigen Maßnahmen des Peacebuilding kaum mehr in Erscheinung tritt. Eine der Ursachen dieser Entwicklung liegt darin, dass die AU zu schwach ist, die selbst gesteckten Ansprüche zu erfüllen. Dies wirft nicht nur die Frage nach einem sinnvollen Aufbau von Kapazitäten auf, sondern auch die nach der Wirksamkeit des zugrundliegenden Modells. Zentrale Aspekte des liberalen Friedensmodells wie eine stabile demokratische Ordnung und die Achtung von Menschenrechten sind jedenfalls in einer Reihe von AU-Mitgliedstaaten unzureichend verwirklicht. Nicht wenige langjährige Staatsoberhäupter sind selbst durch Waffengewalt an die Macht gekommen, auch wenn sie mittlerweile mehrfach wiedergewählt wurden. Wichtige Elemente von Peacebuilding sind hier wie auch in anderen Staaten mit einer Vorgeschichte bewaffneter Konflikte kaum oder sehr begrenzt verinnerlicht. So ist die Schwäche des Profils der APSA bei den Friedensmissionen nicht allein mangelnden Ressourcen geschuldet.

\section{Fazit}

Bei der Politikformulierung, dem Aufbau der Institutionen und den konkreten friedensbezogenen Aktivitäten hat die APSA insgesamt viel erreicht. Sie ist ein Symbol der Emanzipation von äußeren Mächten - allein dies verleiht ihr bereits große Bedeutung. Allerdings sind neue, „afrikanische“ Konzepte und Ansätze noch nicht wirklich erkennbar. Die Strukturen und Operationen der APSA leiden weiterhin unter einem Mangel an Ressourcen. Zudem besteht eine Schieflage im subsidiären Aufbau der APSA und bei der Durchführung von Friedensmissionen, die trotz des Anspruchs eines umfassenden Sicherheitsbegriffs bislang eher eindimensional geblieben sind. Wichtig ist letztlich, dass die AU unter der neuen Kommissionsvorsitzenden Nkosazana Dlamini-Zuma auf eigene politische Konzepte, eine ausgewogene institutionelle Struktur und eine Stärkung des Profils der APSA im operativen Bereich setzt. Allzu oft liegt der Fokus afrikanischer Eliten wie internationaler Geber auf der Funktionalität der APSA und einem eher technisch verstandenen Kapazitätsaufbau. Tatsächlich ist eine stärkere Eigenständigkeit im institutionellen 
wie operativen Bereich notwendig. Ansonsten riskiert die AU im Extremfall, Spielball der Interessen externer Akteure und einiger afrikanischer Eliten zu werden.

Es gibt aber beim Kapazitätsaufbau einige Fallstricke. Einerseits wird dieser immer noch in sehr begrenztem Ausmaß betrieben. Die EU beispielsweise stellt über die Afrikanische Friedensfazilität bislang 100 Millionen Euro bereit, gegenüber den erwähnten 600 Millionen Euro für die afrikanischen Friedensmissionen, die in erster Linie der Kostendeckung dienen. Ein Bericht von 2011 stellte für den Zehnjahresplan zum Kapazitätsaufbau im Rahmen der VN-AU-Kooperation fest, dass oftmals die Linie zwischen dem Aufbau von AU-Kapazitäten durch die VN und der Bereitstellung von Kapazitäten durch die VN ausgesprochen unscharf ist. Außerdem konzentrierten sich VN-Experten mehr auf die Abwicklung von Projekten als auf einen Aufbau von AU-Kapazitäten. ${ }^{20}$ Es ist also fragwürdig, ob „Kapazitätsaufbau drin ist, wo Kapazitätsaufbau draufsteht“.

Andererseits führen auch erfolgreich durchgeführte Maßnahmen nicht automatisch zu „African ownership“ im Bereich Frieden und Sicherheit. Denn die Zukunft der APSA ist nicht nur eine Frage des Geldes und eigener Institutionen, so wenig wie bestehende Defizite einfach nur als Umsetzungsprobleme

20 Security Council Report. 2011. Working Together for Peace and Security in Africa: The Security Council and the AU Peace and Security Council. New York: Security Council Report. Special Research Report: 2011/No. 2, S. 10. abgetan werden sollten. Letztlich ist bislang weiterhin unklar, was „African ownership“ jenseits eigener Strukturen und Aktivitäten im Rahmen der APSA politisch und konzeptionell wirklich meint. Dies führt dazu, dass der institutionelle und operative Bereich auf Annahmen aufbaut, die nicht immer gegeben sind, so die Existenz effektiver regionaler Pfeiler unterhalb der AU und die Verinnerlichung oder jedenfalls Akzeptanz des liberalen Peacebuilding-Ansatzes in den Mitgliedstaaten. Das Engagement von Mitgliedstaaten darf auch nicht einfach auf ihre finanziellen Beiträge oder die Bereitstellung von Truppen reduziert werden, sondern erfordert eine konstruktive Rolle der Länder für Frieden und Sicherheit in ihrem direkten Einflussbereich. Denn davon hängt die Effektivität der regionalen Pfeiler der APSA wie auch der direkten AU-Aktivitäten in den Regionen letztlich ab. Zudem bedarf es einer stärkeren Einbeziehung zivilgesellschaftlicher Akteure aus den Mitgliedstaaten, um neue Ideen und Anstöße in den AU-Prozess einfließen zu lassen und das Projekt der APSA nicht zu einem elitengesteuerten Prozess zu machen. Denn, wie die neue AU-Kommissionsvorsitzende Dlamini-Zuma in ihrer Antrittsrede betonte, die Auswirkung der Politik des Staatenbundes muss daran gemessen werden, wie sie die Bürger Afrikas konkret berührt. ${ }^{21}$

21 Address by the African Union Commission Chairperson Dr. Nkosazana Dlamini Zuma, African Union Headquarters, Addis Ababa, Ethiopia Monday 15 October 2012, http://ccpau.org/wp-content/uploads/2012/10/ Chairperson-Dlamini-Zuma-Handover-Speech-Final-15.10.12.pdf [31. Oktober 2012].

\title{
Testing the Paradigms of Humanitarian Dialogue with Non-State Armed Groups: The Unique Challenges of Ending the Use of Child Soldiers
}

\author{
Janel B. Galvanek and Yvonne Kemper*
}

\begin{abstract}
This article examines the factors and conditions that explain why engagement with NSAGs on child soldiers is particularly challenging. The article identifies the key factors that determine the successful outcome of humanitarian dialogue with NSAGs in general, and then applies this framework to the issue of child soldiers to highlight some of its distinct features. Looking beyond the theoretical analysis, with the help of two specific cases, we will highlight how these unique challenges in ending the use and recruitment of child soldiers can also help explain impasses in dialogue with some of the groups implicated in this offense.
\end{abstract}

Keywords: Child soldiers, children in armed conflict, humanitarian dialogue, non-state armed groups Kindersoldaten, Kinder in bewaffneten Konflikten, humanitärer Dialog, nichtstaatliche bewaffnete Gruppen

\section{Introduction}

A fter seven years in office, Ms. Radhika Coomaraswamy, the outgoing UN Special Representative on Children and Armed Conflict, already had a goal in mind for her successor, namely to end the use of children by state

* Janel B. Galvanek, M.A., M.P.S., is Project Officer and Executive Assistant at the Berghof Foundation in Berlin, Germany. Yvonne Kemper, M.S.F.S., M.P.S., is an independent consultant focusing on human rights and humanitarian affairs. She is based in Boston, Massachusetts. armed forces by 2016. ${ }^{1}$ In contrast, her hopes for successfully engaging with non-state actors on this issue seemed far less ambitious, consisting of "accelerating" the adoption of UN Action Plans, by which these groups would voluntarily commit to end grave violations against children. While engagement with state actors follows well-known rules, Ms. Coomaraswamy's statement acknowledges the complexity of

1 "Radhika Coomaraswamy Speaks at IPI": http://reliefweb.int/report/world/ radhika-coomaraswamy-speaks-ipi-video, accessed on 24 October 2012. 